\title{
Extraction of parathion by hydroxyl-functionalized ionic liquid aqueous two-phase system
}

\author{
Bo Zhou ${ }^{a}$, Gao-zhang Gou, Shi-juan Xu, Na Wu, Wei Liu* \\ Department of Chemistry, College of Science, Honghe University, Mengzi, 661100, China \\ aemail: zhoubo432@163.com, *email:liuwei4758@163.com
}

Keywords: hydroxyl-functionalized ionic liquid; parathion; aqueous two-phase system

\begin{abstract}
An ionic liquid/aqueous two-phase system (ATPS) formed by the hydroxyl-functionalized ionic liquid 3-(2,3-dihydroxypropyl)-1-methylimidazolium chloride ([2,3-dhpmim]Cl) and salt has been developed for extraction of parathion. The experimental parameters affecting the extraction efficiency including the kind of salts, the amounts of the salt, the amount of ionic liquid, extraction times were investigated and optimized. The conclusion shows that the ionic liquid-ATPS can be used for extraction of parathion successfully from environmental water samples. Under optimized conditions, ionic liquid-ATPS has the highest extraction efficiency for parathion (83\%).
\end{abstract}

\section{Introduction}

Organic phosphorus pesticides[1] are insecticides widely used in agricultural activities because of their effective ability to control pests and acarid. They are very difficult to degrade and metabolize. They can be traced in the water, in the soil, in the food and so on. So they not only cause some serious pollution to the environment, but also are harmful to human body. Parathion is one of the most important organic phosphorus. So in this paper, our aim is to detect the amount of parathion in water sample.

The presence of organic phosphorus residues in complicated samples has encouraged the development of various sample preparation techniques[2]. Sample preparation has been identified as the bottleneck of analytical methodologies for the determination of contaminants in complicated samples. Prior sample preparation before instrumental analysis can help to extract, isolate, and concentrate the analytes of interest from complicated matrices including other interfering high molecular mass compounds. Therefore, the current trend of sample preparation techniques is focused on the simplifications, miniaturization, and combination of different steps, such as extraction, concentration, isolation of analytes, clean-up, and instrumental analysis in one single step[3].

Room temperature ionic liquids (RTILs) are entirely composed of organic cations and various anions. Ionic liquids are a class of non-molecular ionic solvents with low melting points. In recent years, ionic liquid have attracted much attention. Compared with water and organic solvents, ILs have many special properties, such as wide liquid range, thermal stability, high ionic conductivity, enhanced solubility with many organic compounds, attractive recyclability, very low flammability, and negligible vapor pressure. Therefore, ILs are considered as environmentally friendly substitutes to conventional organic solvents[4-6].

In this work, we show that organic phosphorus in water sample can be effectively extracted by the ionic liquid two-phase system.

\section{Experimental}

\section{Reagents and solutions.}

O,O-diethyl-O-(p-nitrophenyl) thionophosphate (parathion) standard sample was purchased from Beijing Zhen Xiang Industry \& Trade Co., Ltd.. (Beijing, China). N-methylimidazolium was purchased from Linhai kaile chemical company (Zhejiang Province, China). 1,2-dihydroxy-3-chloropropane was bought from Darui fine chemical company (Shanghai, China). The ionic liquid ([2,3-dhpmim]Cl) was prepared according to the reference[7]. $\mathrm{K}_{2} \mathrm{CO}_{3}, \mathrm{~K}_{3} \mathrm{PO}_{4}$ and 
$\mathrm{K}_{2} \mathrm{HPO}_{4}$ were bought from Guangzhou chemical company (Guangdong Province, China). Other chemicals used were of commercial grade. All reagents were used without further purification. Deionized water was used throughout.

The stock standard solution of parathion $(100 \mathrm{mg} / \mathrm{L})$ was prepared and stored in a refrigerator. The working solution was prepared daily by diluting the mixed standard solution in different concentration using deionized water.

\section{Preparation of LI/salt aqueous two-phase system.}

The phase diagram was prepared according to the turbidimetric titration method. Briefly, a few grams of concentrated IL ([2,3-dhpmim]Cl) solution were weighed into a test tube. Then a solution of known concentration of a salt was added drop-by-drop and mixed. The solution was clear at first. But after a certain amount of the salt was added, one further drop made the mixture turbid and separated into two phases. The mass of the mixture was noted and the composition of the two-phase system was determined at $T=298.15 \mathrm{~K}$ and ambient pressure. Keep adding a few drops of water to the two-phase system, the mixture became clear once again. In order to get sufficient data on the two-phase system, the above procedure was repeated.

\section{Extraction of parathion.}

A proper amount of IL, $\mathrm{K}_{3} \mathrm{PO}_{4}$ and the solution of parathion were added into a graduated glass tube. The volume of the glass tube was calibrated. The mixture was diluted to the mark with deionized water and gently stirred by magnetic stirrer for 30 min which was sufficient to separate the mixture into two liquid phases. The temperature of the systems was controlled at $T=298.15 \mathrm{~K}$ in a thermostated water bath. The phase separation quickly happened after cessation of the stirring process. The volume of top and bottom phases was recorded. Then the sample was collected from the IL-rich upper phase and diluted for analysis. A mass balance check was made between the initial mass of parathion and the amounts in the upper and lower phases on the basis of equilibrium compositions. The relative error in the mass balance was within $2 \%$. To avoid interference from phase components, samples were diluted and analyzed against blanks containing the same phase compositions but without parathion.

High performance liquid chromatography (HPLC) determination.

HPLC determination was carried out on a LC-10AT liquid chromatographic instrument (Shimadzu, Japan) consists of two LC-10ATvp pumps and an SPD-10Avp UV-vis detector set at $254 \mathrm{~nm}$. Data acquisition and process were accomplished with a Chromato-solution Light Workstation (Shimadzu, Japan). The analytical column was a $250 \mathrm{~mm} \times 4.6 \mathrm{~mm}$ i.d. C18 column. The mobile phase was a mixture of acetonitrile and $0.1 \%$ acetic acid $(85: 15, \mathrm{v} / \mathrm{v})$ delivered at a flow rate of $0.8 \mathrm{~mL} / \mathrm{min}$.

\section{Results and disscussion}

\section{Phase diagram.}

Three kinds of salts, such as $\mathrm{K}_{2} \mathrm{HPO}_{4}, \mathrm{~K}_{2} \mathrm{CO}_{3}$ and $\mathrm{K}_{3} \mathrm{PO}_{4}$, had been studied their ability for the formation of ionic liquid aqueous two-phase system. From the figure 1, it was easy to draw the conclusion that the effect of salts for ionic liquid aqueous two-phase system follows the order: $\mathrm{K}_{3} \mathrm{PO}_{4}>\mathrm{K}_{2} \mathrm{HPO}_{4}>\mathrm{K}_{2} \mathrm{CO}_{3}$. At the same time, it indicated that the less salt $\left(\mathrm{K}_{3} \mathrm{PO}_{4}\right)$ was needed to form the ionic liquid-ATPS. So, in this study, $\mathrm{K}_{3} \mathrm{PO}_{4}$ was finally selected for further investigations.

\section{Effect of the amount of K3PO4 on the extraction efficiency.}

In this section, the amount of $\mathrm{K}_{3} \mathrm{PO}_{4}$ on the extraction efficiency of parathion was studied. 2.0 $\mathrm{mL} \mathrm{K}_{3} \mathrm{PO}_{4}$ solution with different concentrations (5\% to $35 \%$ ) was added to system while the amounts of ionic liquid and parathion were fixed. The results were showed in the figure 2 . From the figure 2, the extraction efficiency of parathion increased with the increase of $\mathrm{K}_{3} \mathrm{PO}_{4}$ concentration from $5 \%$ to $25 \%$. When the concentration exceeded $25 \%$, the extraction efficiency did not increase obviously. So, the optimal concentration of $\mathrm{K}_{3} \mathrm{PO}_{4}$ solution was $25 \%$. 


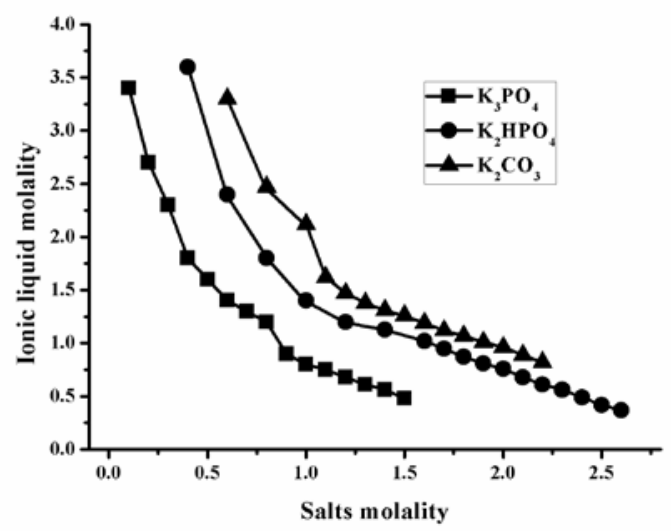

Figure 1 Phase diagram of IL/Salts aqueous two-phase system

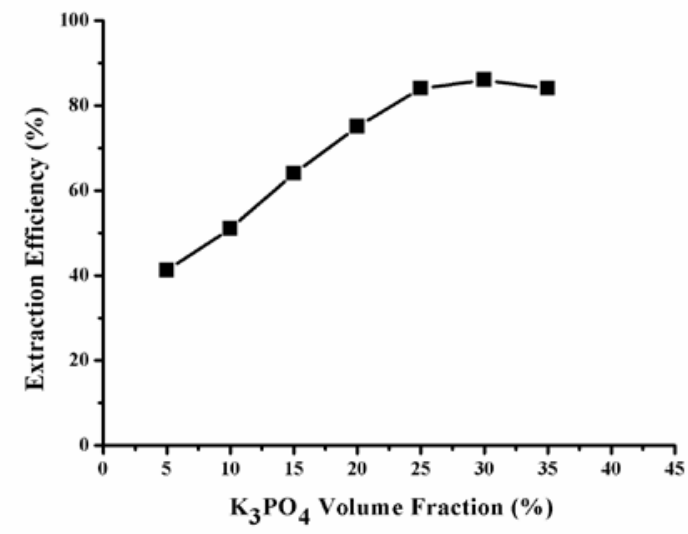

Figure 2 Effect of $\mathrm{K}_{3} \mathrm{PO}_{4}$ concentration on the extraction efficiency

\section{Effect of the amount of ionic liquid.}

The amount of ionic liquid has an important effect on the extraction efficiency too. Different amounts of ionic liquid were added to systems while the amounts of $\mathrm{Na}_{3} \mathrm{PO}_{4}$ and parathion were fixed respectively. The influence of the amount of ionic liquid on the extraction efficiency was demonstrated in the figure 3. The extraction efficiency increased obviously at the beginning. When the amount of ionic liquid was higher than $2.0 \mathrm{~mL}$, the extraction efficiency increased very slightly. So, the optimal amount of ionic liquid was $2.0 \mathrm{~mL}$.

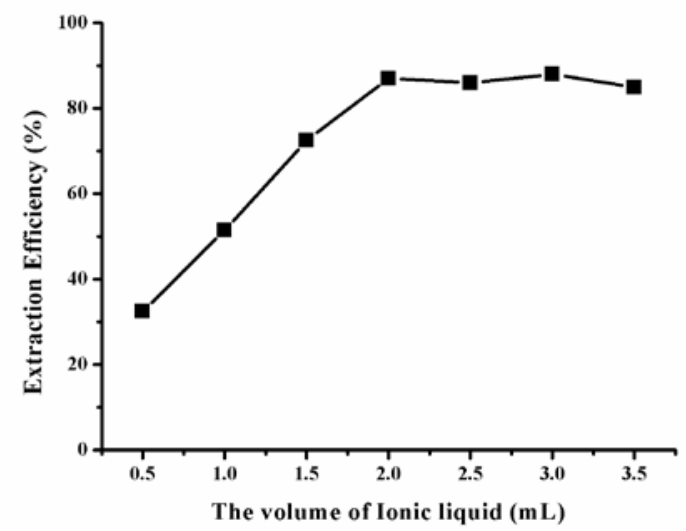

Figure 3 Effect of ionic liquid amount on the extraction efficiency

\section{Effect of extraction time.}

Extraction time is one of the essential factors on extraction efficiency. In the work, experiments were performed with extraction time from 5 to 30 min to obtain the optimum condition. The results were shown in figure 4. According to results, the enrichment factor of parathion was raised as the extraction time increased. When the time was greater than $20 \mathrm{~min}$, the enrichment factor didn't 
change almost. So, 20 min was used as the optimal condition for the procedure.

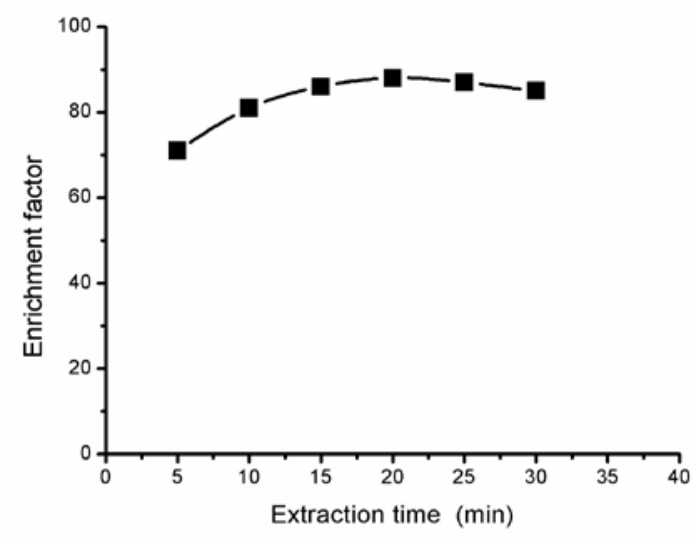

Figure 4 Effect of extraction time on the enrichment factor

\section{Conclusion}

In this work, a simple, fast and sensitive analytical method using hydroxyl-functionalized ionic liquid aqueous two-phase system extraction followed by high performance liquid chromatography has been studied for parathion in water samples. With the concentration of $\mathrm{K}_{3} \mathrm{PO}_{4} 25 \%$ (mass fraction), ionic liquid $2.0 \mathrm{~mL}$ and extraction time $20 \mathrm{~min}$, ionic liquid-ATPS had the highest extraction efficiency for parathion in water sample (87\%).

\section{Acknowledgements}

This work was supported by the Science Research Foundation of Education Department of Yunnan province (No. 2013FZ121), the Chemistry of Key Construction Disciplines for Master Degree Program of Yunnan (No.HXZ1303) and the PhD research startup foundation of Honghe university (No.14bs21).

\section{Reference}

[1]Giuseppe Cinelli, Pasquale Avino, Ivan Notardonato, Mario Vincenzo Russo. Ultrasound-vortex-assisted dispersive liquid-liquid microextraction coupled with chromatography with a nitrogen-phosphorus detector for simultaneous and rapid determination of organophosphorus pesticides and triazines. [J]. Analytical Methods, 2014(6)782-790.

[2] Marco Scheurer, Stefanie Heß, Frauke Lüddeke, Frank Sacher, Hans Güde, Herbert Löffler and Claudia Gallert. Removal of micropollutants, facultative pathogenic and antibiotic resistant bacteria in a full-scale retention soil filter receiving combined sewer overflow. [J]. Environmental Science: Processes \& Impacts, 2015(17)186-196.

[3] Dan Lu, Yuan Yang, Xiaofei Luo, Chengjun Sun. A fast and easy GC-MS/MS method for simultaneous analysis of 73 pesticide residues in vegetables and furits. [J]. Analytical Methods, 2013(50)1721-1732.

[4] Menghao Zhu, Junmei Zhao, Yingbo Li, Nada Mehio, Yuruo Qi, Huizhou Liu, Sheng Dai. An ionic liquid-based synergistic extraction strategy for rare earths. [J]. Green Chemistry, 2015(17)2981-2993.

[5] Li Huaxi, Li Zhuo, Yin Jingmei, Li Changping, Chi Yansheng, Liu Qingshan, Zhang Xiuling, Welz-Biermann Urs. Liquid-liquid extraction process of amino acids by a new amide-based 
functionalized ionic liquid. [J]. Green Chemistry, 2012(14)1721-1717.

[6] Mor Sendovski, Netta Nir, Ayelet Fishman. Bioproduction of 2-phenylethanol in a biphasic ionic liquid aqueous system. [J]. Journal of Agricultural and Food Chemistry, 2010(58)2260-2265.

[7] Yue Qin Cai, Ye Liu, Guo Hua Gao. Synthesis and application of an IL-supported diol as protecting group for aldehydes. [J]. Chinese Chemical Letters, 2007(18)1205-1208. 